\title{
Effective action for reggeized gluons, classical gluon field of relativistic color charge and color glass condensate approach
}

\author{
S. Bondarenko ${ }^{1, a}$, L. Lipatov ${ }^{2,3}$, A. Prygarin ${ }^{1}$ \\ ${ }^{1}$ Physics Department, Ariel University, Ariel, Israel \\ 2 St.Petersburg Nuclear Physics Institute, Saint Petersburg, Russia \\ ${ }^{3}$ II Institute of Theoretical Physics, Hamburg University, Hamburg, Germany
}

Received: 1 June 2017 / Accepted: 24 July 2017 / Published online: 8 August 2017

(C) The Author(s) 2017. This article is an open access publication

\begin{abstract}
We discuss application of formalism of small$x$ effective action for reggeized gluons (Gribov, Sov. Phys. JETP 26:414, 1968; Lipatov, Nucl. Phys. B 452:369, 1995; Lipatov, Phys. Rep. 286:131, 1997; Lipatov, Subnucl. Ser. 49:131, 2013; Lipatov, Int. J. Mod. Phys. Conf. Ser. 39: 1560082, 2015; Lipatov, Int. J. Mod. Phys. A 31(28/29): 1645011, 2016; Lipatov, EPJ Web Conf. 125:01010, 2016; Lipatov, Sov. J. Nucl. Phys. 23:338, 1976; Kuraev et al., Sov. Phys. JETP 45:199, 1977; Kuraev et al., Zh Eksp, Teor. Fiz. 72:377, 1977; Balitsky and Lipatov, Sov. J. Nucl. Phys. 28:822, 1978; Balitsky and Lipatov, Yad. Fiz. 28:1597 1978), for the calculation of classical gluon field of relativistic color charge, similarly to that done in CGC approach of McLerran and Venugopalan, Phys. Rev. D 49:2233 (1994), JalilianMarian et al., Phys. Rev. D 55:5414 (1997), Jalilian-Marian et al., Nucl. Phys. B 504:415 (1997), Jalilian-Marian et al., Phys. Rev. D 59:014014 (1998), Jalilian-Marian et al., Phys. Rev. D 59:014015 (1998), Iancu et al., Nucl. Phys. A 692:583 (2001), Iancu et al., Phys. Lett. B 510:133 (2001), Ferreiro et al., Nucl. Phys. A 703:489 (2002). The equations of motion with the reggeon fields are solved in LO and NLO approximations and new solutions are found. The results are compared to the calculations performed in the CGC framework and it is demonstrated that the LO CGC results for the classical field are reproduced in our calculations. Possible applications of the NLO solution in the effective action and CGC frameworks are discussed as well.
\end{abstract}

\section{Introduction}

In the framework of perturbative QCD, the calculations of classical field created by a relativistic color charge is an important task and its results are useful in many physical applications. There are self-consistent approaches for the cal-

\footnotetext{
a e-mail: sergeyb@ariel.ac.il
}

culation of the mean field in the framework of Wong's equations, in the theory of non-Abelian plasma physics and in the classical and quantum transport theories, see [25-34] and references therein. In the context of the Color Glass Condensate (CGC) approach, the knowledge of solution of the equations of motion is an important initial set up for the further small- $x$ evolution of the gluonic system, see [13-20].

The CGC approach is based on the renormalization group (quantum evolution) applied to some initial classical configuration of the gluon field. Equations of motion for the gluon field in this case are derived within some effective action, see details in [13-20]. An important ingredient of the framework is a source of the gluon field, see [14-20] and discussion further in the paper. The structure of this term, in turn, determines the form of equations of motion and correspondingly the form of solution of the equations, which are known to the LO precision. The Balitsky, Fadin, Kuraev, Lipatov (BFKL) like, [8-12], small- $x$ behavior of the gluon density, a non-linear Gribov, Levin, Ryskin (GLR) equation, see [21] as well as Balitsky-Kovchegov (BK) like, [35-37], corrections to this density were reproduced in the framework of this approach.

In the frameworks based on the theory of BFKL Pomeron, [2-12], the source terms in the action are considered as well, see [38-44]. There the Pomeron is coupled to the source, it is usual formulation of different RFT theories, see [45-50]. The effective action approach of [2-7] can be considered as some generalization of Gribov's Regge calculus, [1], for the case of QCD degrees of freedom. Besides the usual gluon field, it includes two additional reggeon fields and widely used for the calculations of different quasi-elastic LO and NLO production amplitudes in the multi-Regge kinematics, see [51-60], or calculation of NLO corrections to the BKP, $[61,62]$, kernel, see [63].

In our paper, we use the effective action for reggeized gluons exploring ideas of [2-7]. Using light-cone gauge, we 
consider a problem with only one longitudinal gluon field in the equations of motion included. With the two reggeon fields present in the approach, the first reggeon field is defined as a LO value of the corresponding gluon field, whereas the second reggeon field arises as a source term in the Lagrangian. The form of the effective currents, arising in the equations of motion, therefore, can be obtained or directly from the effective action expression from [2-7] or from the self-consistency conditions for the solution of the equations of motion, in both cases we obtain the same structure of the current. Respectively, in the next Sect. 2, we discuss the set up of the problem, i.e. we consider the expression for the effective action, further, in the Sect. 3, we write equations of motion for the gluon fields of the problem in light-cone gauge. In the Sect. 4 we solve these equations with the LO accuracy and demonstrate that obtained solution is the same as obtained in CGC approach in the limit of zero reggeon field. Section 5 is dedicated to the semi-classical Reggeon action obtained from the initial one and in the Sect. 6 we discuss the similarities and differences between the solutions obtained in the paper and in the framework of CGC approach. The Conclusion is presented in Sect. 7, a calculation of the NLO solution is in the Appendix $\mathrm{C}$ and in the Appendixes $\mathrm{A}$ and $\mathrm{B}$ we discuss the form of the current in the effective action for reggeized gluon approach.

\section{Effective action for reggeized gluons with color field source}

The effective action, see [2-7], is a non-linear gauge invariant action which correctly reproduces the production of the particles in direct channels at a quasi-multi-Regge kinematics. It is written for the local in rapidity interactions of physical gluons in direct channels inside of some rapidity interval $(y-\eta / 2, y+\eta / 2)$. In turn, the interaction between the different clusters of gluons at different but close rapidities can be described with the help of reggeized gluon fields ${ }^{1} A_{-}$and $A_{+}$ interacting in crossing channels. Those interaction are nonlocal in rapidity space. This non-local term is not included in the action, the term of interaction between the reggeon fields in the action is local in rapidity and can be considered as some kind of renormalization term in the Lagrangian. The action is gauge invariant and written in the covariant form in terms of gluon field $v$ as

$$
\begin{aligned}
S_{\text {eff }}= & -\int d^{4} x\left(\frac{1}{4} G_{\mu \nu}^{a} G_{a}^{\mu \nu}+\operatorname{tr}\left[\left(A_{+}\left(v_{+}\right)\right.\right.\right. \\
& \left.\left.\left.-A_{+}\right) j_{\text {reg }}^{+}+\left(A_{-}\left(v_{-}\right)-A_{-}\right) j_{\text {reg }}^{-}\right]\right),
\end{aligned}
$$

\footnotetext{
1 We use the Kogut-Soper convention for the light-cone for the lightcone definitions with $x_{ \pm}=\left(x_{0} \pm x_{3}\right) / \sqrt{2}$ and $x_{ \pm}=x^{\mp}$.
}

where

$A_{ \pm}\left(v_{ \pm}\right)=\frac{1}{g} \partial_{ \pm} O\left(x^{ \pm}, v_{ \pm}\right)=v_{ \pm} O\left(x^{ \pm}, v_{ \pm}\right)$

with $O\left(x^{ \pm}, v_{ \pm}\right)$as some operators, see [2-7], Appendix A, Appendix B and Sect. 4 in Iancu et al. [14-20]. The form of reggeon current we take is the following: ${ }^{2}$

$j_{\mathrm{reg} a}^{ \pm}=\frac{1}{C(R)} \partial_{i}^{2} A_{a}^{ \pm}$

where $C(R)$ is the eigenvalue of Casimir operator in the representation $\mathrm{R}, C(R)=N$ in the case of adjoint representation used in the paper. There are additional kinematical constraints for the reggeon fields

$\partial_{-} A_{+}=\partial_{+} A_{-}=0$

corresponding to the strong-ordering Sudakov components in the multi-Regge kinematics, see [2-7]. Here, as usual, $\partial_{i}$ denotes the derivative on transverse coordinates. In the framework with an external source of the color charge introduced, keeping only gluon field depending terms in the Eq. (1) action, we rewrite Eq. (1) as

$S_{\mathrm{eff}}=-\int d^{4} x\left(\frac{1}{4} G_{\mu \nu}^{a} G_{a}^{\mu \nu}+v_{-} J^{-}\left(v_{-}\right)+v_{+} J^{+}\left(v_{+}\right)\right)$,

with

$J^{ \pm}\left(v_{ \pm}\right)=O\left(v_{ \pm}\right) j_{\text {reg }}^{ \pm}$

Under variation on the gluon fields these currents reproduce the Lipatov's induced currents

$\delta\left(v_{ \pm} J^{ \pm}\left(v_{ \pm}\right)\right)=\left(\delta v_{ \pm}\right) j_{\mp}^{\text {ind }}\left(v_{ \pm}\right)=\left(\delta v_{ \pm}\right) j^{ \pm}\left(v_{ \pm}\right)$

with shortness notation $j_{\mp}^{\text {ind }}=j^{ \pm}$introduced. This current posseses a covariant conservation property:

$\left(D_{ \pm} j_{\mp}^{\text {ind }}\left(v_{ \pm}\right)\right)^{a}=\left(D_{ \pm} j^{ \pm}\left(v_{ \pm}\right)\right)^{a}=0$.

Here and further we denote the induced current in the component form in the adjoint representation ${ }^{3}$ as

$$
\begin{aligned}
j_{a}^{ \pm}\left(v_{ \pm}\right) & =-\imath \operatorname{tr}\left[T_{a} j^{ \pm}\left(v_{ \pm}\right)\right] \\
& =\frac{1}{N} \operatorname{tr}\left[f_{a} O f_{b} O^{T}\right]\left(\partial_{i}^{2} A_{\mp}^{b}\right),
\end{aligned}
$$

\footnotetext{
2 It is rewritten in comparison to the current from [2-7].

3 We use $\left(T_{a}\right)_{b c}=-\imath f_{a b c}$ definition of the matrices and write only "external" indexes of the $f_{a b c}=\left(f_{a}\right)_{b c}$ matrix in the trace.
} 
see Appendices A and B. It will be shown in the following sections, that the requests of self-consistency of the solution of equations of motion will lead independently to the Eqs. (6)-(8) form and described properties of the current. Applying the light-cone gauge $v_{-}=0$, the second current term in the r.h.s. of Eq. (5) looks equal to zero on the first sight, but due the $x_{-}$derivative in the Eq. (2) this term acquires some non-zero value on the boundaries of integration, see also discussion in $[22,23]$. For our calculations these contributions are not important, but it is interesting to note, that after the integration on $x_{ \pm}$variables in the effective currents terms in the Lagrangian, the remaining expressions can be interpreted as distribution of the light-cone straight lines in the three dimensional space with the probabilities of distribution determined by the reggeon fields. This geometrical picture potentially can be very interesting, because a deformation of this lines can lead to some interconnections of the approach with framework of [24].

\section{Equations of motion in light-cone gauge}

The classical equations of motion for the gluon field $v_{\mu}$ field which arose from the Eq. (5) action are the following:

$$
\left(D_{\mu} G^{\mu \nu}\right)_{a}=\partial_{\mu} G_{a}^{\mu \nu}+g f_{a b c} v_{\mu}^{b} G^{c \mu \nu}=j_{a}^{+} \delta^{\nu+}+j_{a}^{-} \delta^{\nu-}
$$

We assume also, that the form of the currents in Eq. (10) is not fixed yet, it will be demonstrated that it can be independently determined on the base of self-consistency request applied for the solutions of equations of motion, see Eq. (29) in the next section. Of course, this resulting current will be the same as the induced current Eq. (9) introduced in the previous Section.

The light-cone gauge $v_{-}^{a}=0$ is applied further in the equations of motion and thereafter in the final effective action as well. Considering Eq. (10) we obtain the following equations for the different field components.

1. The variation of the action with respect to $v_{+}^{a}$ gives:

$$
-\partial_{i} \partial_{-} v_{a}^{i}-\partial^{+} \partial_{-} v_{a+}-g f_{a b c} v_{i}^{b}\left(\partial_{-} v^{i c}\right)=j_{a}^{+}\left(v_{+}\right),
$$

or

$$
-\left(D_{i}\left(\partial_{-} v^{i}\right)\right)_{a}-\partial_{-}^{2} v_{a+}=j_{a}^{+}\left(v_{+}\right)
$$

2. The variation on field $v_{-}^{a}$ provides

$$
\partial_{\mu} G_{a}^{\mu-}+g f_{a b c} v_{\mu}^{b} G^{c \mu-}=j_{a}^{-}\left(v_{-}\right),
$$

and, requesting the following current's property $j_{a}^{-}\left(v_{-}=\right.$ $0)=-\partial_{i}^{2} A_{a+}$, we obtain:

$$
\left(D^{-} G_{-+}\right)_{a}+\left(D^{i} G_{i+}\right)_{a}=-\partial_{i}^{2} A_{a+}
$$

It can be written as following:

$$
\begin{aligned}
\partial_{+} & \partial_{-} v_{a+}+g f_{a b c} v_{+}^{b}\left(\partial_{-} v_{+}^{c}\right) \\
& +\partial^{i}\left(\partial_{i} v_{a+}-\partial_{+} v_{a i}+g f_{a b c} v_{i}^{b} v_{+}^{c}\right) \\
& +g f_{a b c} v^{b i}\left(\partial_{i} v_{+}^{c}-\partial_{+} v_{i}^{c}+g f^{c d f} v_{d i} v_{f+}\right) \\
= & -\partial_{i}^{2} A_{a+},
\end{aligned}
$$

and presented in one of the forms:

$$
\begin{aligned}
& \left(D_{+}\left[v_{+}\right]\left(\partial_{-} V_{+}\right)\right)_{a}+\left(D^{i}\left(\partial_{i} V_{+}\right)\right)_{a}-\partial^{i}\left(D_{+} V_{i}\right)_{a} \\
& -g f_{a b c} v^{b i}\left(D_{+} V_{i}\right)^{c}=-\partial_{i}^{2} A_{a+}
\end{aligned}
$$

or

$$
\begin{aligned}
& \left(D_{+}\left[v_{+}\right]\left(\partial_{-} v_{+}\right)\right)_{a}+\left(D^{i}\left(\partial_{i} v_{+}\right)\right)_{a} \\
& -\left(D^{i}\left[v_{i}\right]\left(D_{+}\left[v_{+}\right] v_{i}\right)\right)_{a}=-\partial_{i}^{2} A_{a+},
\end{aligned}
$$

see Appendix $\mathrm{C}$ for more details.

3. The variation on field $v_{\perp}^{a}$ gives in turn:

$$
\left(D^{+} G_{+i}\right)_{a}+\left(D^{-} G_{-i}\right)_{a}+\left(D^{j} G_{j i}\right)_{a}=0 .
$$

Rewriting this equation as

$$
\begin{gathered}
\partial_{-}\left(\partial_{+} v_{a i}-\partial_{i} v_{a+}+g f_{a b c} v_{+}^{b} v_{i}^{c}\right)+\partial_{+} \partial_{-} v_{a i} \\
\quad+g f_{a b c} v_{+}^{b}\left(\partial_{-} v_{i}^{c}\right)+\left(D^{j} G_{j i}\right)_{a}=0 .
\end{gathered}
$$

we finally obtain:

$$
2\left(D_{+}\left(\partial_{-} V_{i}\right)\right)_{a}-\left(D_{i}\left(\partial_{-} V_{+}\right)\right)_{a}+\left(D^{j} G_{j i}\right)_{a}=0 .
$$

The LO perturbative solutions of these equations are presented in the next section.

\section{The LO solution: from effective action approach to CGC expressions}

For the action without the external source, at $j_{a}^{+}=0$, the trivial solution at the first perturbative order can be easily obtained: 
$v_{\perp}=0, \quad v_{+}=A_{+}$,

see [2-7]. At the presence of an external source the solution is changing and the following ansatz as solution of Eqs. (11)(20) can be introduced:

$v_{-}=0, v_{\perp}=\Lambda\left[g A_{+}\right]+g \Lambda_{1}\left[g A_{+}\right]+\cdots$,

$v_{+}=A_{+}+g \Phi\left[g A_{+}, v_{\perp}\right]+\cdots, \partial_{-} A_{+}=0$,

with still unknown form of the external current, which properties we will establish from the requests of self-consistency of the solution. We note, that this ansatz is valid for the large values of reggeon field when $g A_{+} \sim 1$ and in principal it can be useful for the situations of non-symmetrical scattering processes such as DIS or scattering off nuclei.

1. We begin from Eq. (20) taking there $\partial_{-} V_{+}=0$, see Eqs. (4) and (22). We will see further that at LO $G_{i j}=0$, therefore the equation reads as

$\left(D_{+}\left(\partial_{-} V_{i}\right)\right)_{a}=0$

It's solution has the following form

$\partial_{-} v_{i}^{b}=U^{b c}\left(v_{+}\right) \partial_{-} \rho_{c i}\left(x^{-}, x_{\perp}\right)$,

with $U^{b c}$ as some function which form is determined by the request that it's covariant derivative is equal to zero. The form of the function $\rho_{c i}\left(x^{-}, x_{\perp}\right)$ is arbitrary in this case, it is proportional to the color charge density in the CGC approach. With LO precision, in the sense of Eq. (22) ansatz, we obtain:

$v_{i}^{b}=U^{b c}\left(v_{+}\right) \rho_{c i}\left(x^{-}, x_{\perp}\right)$.

The form of $U^{a b}$ function in Eq. (25) can be written consistently with the used in [14-20]:

$$
\begin{aligned}
v_{i}^{b} & =\operatorname{tr}\left[f^{b} U_{-\infty, x^{+}}\left(v_{+}\right) f^{c} U_{x^{+}, \infty}\left(v_{+}\right)\right] \rho_{c i}\left(x^{-}, x_{\perp}\right) \\
& =U^{b c} \rho_{c i}\left(x^{-}, x_{\perp}\right)
\end{aligned}
$$

where $U_{-\infty, x^{+}}=P e^{g \int_{-\infty}^{x^{+}} d x^{\prime}+v_{+}^{a}}$ and which correspond to the form of the induced current in the effective action when $O$ operator is taken in the form of simple ordered exponential, see Eq. (9) and Appendices A and B.

2. The leading order solution of Eq. (12)

$-D_{i} \partial_{-} v_{a}^{i}=j_{a}^{+}$

will determine the form of unknown function $\rho_{c i}$ $\left(x^{-}, x_{\perp}\right)$ in terms of the given external current ${ }^{4} j_{a}^{+}$,

\footnotetext{
${ }^{4}$ In the given framework we can take 1.h.s. of Eq. (27) as definition of the external current.
}

assumed to be unknown for the moment. Taking Eq. (27) to LO, we obtain:

$-\partial_{i} \partial_{-} v_{a}^{i}=j_{a}^{+}$

The current in the r.h.s. of Eq. (28) we write in the form self-consistent with Eq. (26):

$j_{a}^{+}=-U^{a b}\left(v_{+}\right) \tilde{J}_{b}^{+}\left(x^{-}, x_{\perp}\right)$,

we see, that this condition of self-consistency dictates the same form of the current as induced current introduced in Eq. (7), see Eq. (9). Now, we have to the first perturbative order:

$\partial_{i} \partial_{-} \rho_{a}^{i}=\tilde{J}_{a}^{+}\left(x^{-}, x_{\perp}\right)$,

which is the same equation as in [14-20]. The Eq. (30) has no simple solution and, following to [14-20], we assume the following structure of this term:

$\tilde{J}_{a}^{+}\left(x^{-}, x_{\perp}\right)=\partial_{i} \partial_{-} \tilde{j}_{a}^{i}\left(x^{-}, x_{\perp}\right)$,

that gives

$\rho_{c}^{i}\left(x^{-}, x_{\perp}\right)=\tilde{j}_{c}^{i}\left(x^{-}, x_{\perp}\right)$.

In CGC approach the another assumption is done, namely it is assumed in [13-20] that

$\partial_{-} \tilde{j}_{a}^{i}\left(x^{-}, x_{\perp}\right)=\delta\left(x^{-}\right) \tilde{\rho}_{c}^{i}\left(x_{\perp}\right)$,

with some known $\tilde{\rho}_{c}^{i}\left(x_{\perp}\right)$ functions. We note, that this assumption providing the factorization between $x^{-}$and $x_{\perp}$ coordinates in the LO leads to the difficulties in the NLO solution, it is seen already from Eq. (12). Still, accepting this assumption, we obtain:

$\tilde{j}_{c}^{i}\left(x^{-}, x_{\perp}\right)=\theta\left(x^{-}\right) \tilde{\rho}_{c}^{i}\left(x_{\perp}\right)$,

that provides

$\rho_{c}^{i}\left(x^{-}, x_{\perp}\right)=\theta\left(x^{-}\right) \rho_{c}^{i}\left(x_{\perp}\right)=\theta\left(x^{-}\right) \tilde{\rho}_{c}^{i}\left(x_{\perp}\right)$

and correspondingly

$v_{i}^{b}\left(x^{+}, x_{\perp}\right)=\theta\left(x^{-}\right) U^{b c}\left(v_{+}\right) \rho_{c i}\left(x_{\perp}\right)$,

where the first term of perturbative expansion of Eq. (36) is in full agreement with the LO CGC result of [14-20].

3. For the last equation of motion, Eq. (15), we have at the first order

$\partial_{i} \partial^{i} V_{a+}=-\partial_{i}^{2} A_{a+}$, 
that gives

$v_{a+}=A_{a+}$,

in correspondence with Eq. (22), see [2-7]. In turn, it provides the first order solution for the field $v_{i}$ :

$$
v_{i}^{b}\left(x^{+}, x_{\perp}\right)=U^{b c}\left(A_{+}\right) \rho_{c i}\left(x^{-}, x_{\perp}\right) .
$$

We underlie, that this solution consists all orders of $g$ through the ordered exponential $U^{b c}$, that is the novel result of our calculations.

In order to relate obtained LO solutions Eqs. (38)-(39) with CGC results, we note, that the results of [14-20] for the classical gluon field are reproduced taking $A_{+}=0$ in these expressions.

\section{LO structure of the effective action}

Calculations in the previous section we can consider as a formulation of RFT calculus based on the effective action approach. In this case, basing on the Eq. (A.8) for the current, we consider solution of equations of motion as solution for the classical gluon field in the presence of $A_{-}$source. This transition can be done by the following substitution:

$\tilde{J}_{a}^{+} \rightarrow-\partial_{\perp}^{2} A_{a}^{+}$,

see Eq. (A.8) again and discussions in [14-20] and [22,23]. Therefore, instead of Eq. (30), we obtain:

$\partial_{i} \partial_{-} \rho_{a}^{i}=-\frac{1}{N} \partial_{\perp}^{2} A_{a}^{+}$,

or

$\rho_{a}^{i}=\frac{1}{N} \partial_{-}^{-1}\left(\partial^{i} A_{-}^{a}\right)$,

where the condition $G_{i j}=0$ is provided at LO approximation. Correspondingly, all results of the previous section can be rewritten and we obtain for Eq. (26):

$v_{i}^{a}=\frac{1}{N} U^{a b}\left(v_{+}\right)\left(\partial_{-}^{-1}\left(\partial_{i} A_{-}^{b}\right)\right)$.

The Eq. (38) solution for field $v_{+}$remains unchanged under substitution Eq. (42) as well as form of the NLO solution of Appendix C.

Inserting obtained classical gluon fields solutions in the Eq. (1) action, we will obtain a action which will depend only on the reggeon fields, see [2-7], determining the LO RFT action of the approach. Formally, due to the presence of ordered path exponential in the solutions, the action will includes all order perturbative terms which can be important for large $v_{+} \approx A_{+}$in the processes where some large color charge is created. The expansion of these exponential must be supplemented by solution of equations of motion to corresponding orders, otherwise only part of the usual perturbative corrections will be accounted. In general, the following expansion for the action exists:

$S_{\mathrm{eff}}=-\int d^{4} x\left(s_{1}\left[g, A_{+}, A_{-}\right]+g s_{2}\left[g, A_{+}, A_{-}\right]+\cdots\right)$,

where additional dependence on the coupling constants in the different terms of the Lagrangian is arising through the ordered exponentials in the classical solutions for the gluon field accordingly to the ansatz of Eq. (22).

In order to calculate this action we need to know the components of the field strength tensor, with LO precision we have:

$G_{+-}^{a}=0, G_{i+}^{a}=\partial_{i} A_{+}^{a}, G_{i-}^{a}=-\partial_{-} v_{i}^{a}, G_{i j}=0$,

that gives

$$
\frac{1}{4} G_{\mu \nu} G^{\mu \nu}=-G_{i-} G_{i+}=\left(\partial_{-} v_{i}^{a}\right) \cdot\left(\partial_{i} A_{+}^{a}\right) .
$$

Therefore, for the Eq. (5) effective action we obtain to LO:

$$
\begin{aligned}
S_{\mathrm{eff}}= & -\int d^{4} x\left(\left(\partial_{i} A_{+}^{a}\right) U^{a b}\left(A_{+}\right)\left(\partial_{-} \rho_{i} b\left(x_{\perp}\right)\right)\right. \\
& \left.+\frac{1}{N} A_{+}^{a}\left(O^{a b}\left(A_{+}\right)+N \delta^{a b}\right)\left(\partial_{\perp}^{2} A_{-}^{b}\right)\right),
\end{aligned}
$$

with $O^{a b}\left(A_{+}\right)=\operatorname{Tr}\left[f^{a} O\left(A_{+}\right) f^{b}\right]$ in adjoint representaion. Using Eq. (42) we rewrite this expression as

$$
\begin{aligned}
S_{\mathrm{eff}}= & -\frac{1}{N} \int d^{4} x\left(\left(\partial_{i} A_{+}^{a}\right) U^{a b}\left(A_{+}\right)\left(\partial_{i} A_{-}^{b}\right)\right. \\
& \left.+A_{+}^{a}\left(O^{a b}\left(A_{+}\right)+N \delta^{a b}\right)\left(\partial_{\perp}^{2} A_{-}^{b}\right)\right) .
\end{aligned}
$$

We obtained, that due the ordered exponential in the action, there are some additional corrections which were not considered in [2-7]. However, obtained in expression Eq. (48) corrections are not complete, we also need the higher order solutions of equations of motion. Thus, using results of Appendix $\mathrm{C}$, we can calculate full tree NLO corrections to the reggeonreggeon transition vertices as well, but we postpone this task for the following publications. 


\section{Effective action for reggeized gluons and CGC approach}

In the CGC approach, auxiliary soft and semi-hard gluon fields are added to classical gluon field. Integrating the semihard fields out, some effective action is obtained and relations similar to Eqs. (38), (39) are used there as an initial condition for the further small-x evolution of the gluon density operator. Therefore, it will be constructive to determine the counterpart to the reggeon field in the CGC approach. From [14-20] we know, that the classical gluon field $v_{+\mathrm{cl}}^{a}$ is zero there and only the fluctuations of this field are considered

$v_{+\mathrm{cl}}=\delta v_{+}+a_{+}$,

which are ordered in the longitudinal momenta. There are semi-hard and soft fluctuations of the field, $\delta v_{+}$and $a_{+}$correspondingly, see details in [14-20]. By direct comparison of this field's representation with Eq. (38), we see that the $\delta v_{+}$ fluctuation in the CGC is precisely $A_{+}$reggeon field in the effective action, see also kinematic properties of the field in $[2-7,14-20]$. The difference between two approaches is that whereas the reggeon fields are present in the effective action initially as some parameter of the problem, which must be considered separately after all in the path integral as independent fields, in the CGC approach the reggeon like field $\delta v_{+}$ appears as fluctuation around $v_{+\mathrm{cl}}^{a}=0$ classical solution and integrated out. As we obtained above, taking $A_{+}=0$ in the Eqs. (26) and (38) we will reproduce the CGC answers for the LO classical fields configuration. The counterpart of gluon density operator (source) in the CGC calculations is $A_{-}$, the second reggeon field, see Eqs. (41)-(42), that allows to relate results of the approaches. We also note, that the classical gluon field Eq. (25) consists terms to all order of coupling constant, that differ it from the classical solutions considered in the CGC approach.

There is an important point that requires further clarification. Expansion of the action in terms of background field (reggeon field or $\delta v_{+}$fluctuation) requires solution of equations of motion for gluon fields at the same order of background field. Namely, taking non-zero $\delta v_{+}$we will obtain that the NLO solution for the gluon field will depend on $\delta v_{+}$ already, see Appendix C. Thereby, expanding the Lagrangian in terms of background fields, we have to account the same order terms which arise also from the square of field strength tensor and from the currents in the action. Indeed, there is NLO solution of equations of motion, see Appendix C, which after the insertion into the Lagrangian will reproduce the same order terms as in the expansion of the effective currents in respect to the soft fluctuations, these corrections are absent in CGC approach, see also $[22,23,64,65]$ where the NLO correction to the CGC framework results were discussed.
We also note, that both approaches give the similar equations at the level of equations of motion, when the simplest form of $O$ and $O^{T}$ operators are used, see Appendices B and $\mathrm{C}$. This is related to the fact that the additional term in the CGC action can be considered as resulting from the integration on $x_{-}$coordinate of the current term in the effective action for reggeized gluons, see Iancu et al. in [14-20] and $[22,23]$ for the relevant discussions.

\section{Conclusion}

In this paper we consider application of the effective action approach for reggeized gluons to the calculation of a classical gluon field produced by relativistic color charge. We demonstrate, that effective action for reggeized gluons can be obtained from QCD action, when both reggeon fields are introduced as non-zero LO solutions for the classical longitudinal gluon fields and as sources of each other. The form of effective currents in the action in this case, can be obtained from the request of self-consistency of classical equations of motion. We also obtained Eqs. (38), (39), (C.15) and (C.22) expressions for the classical gluon fields calculated in the effective action formalism, which consist all order terms in respect to the coupling constant, this is a main result of the paper. In these calculations the results for the classical gluon fields of CGC approach can be reproduced in the limit of zero reggeon fields, see discussion in the previous Section.

In the framework of the effective action, the performed calculations can be considered as solution of classical equations of motion with the reggeons fields introduced as LO classical solutions of longitudinal gluon fields. Considering field $A_{-}$in the as an external source, see Eqs. (41)-(42), the same problem can be understood as calculation of some effective particle-reggeon-particle vertex, see expression Eq. (5). In this case, introducing fluctuations around the classical solution and integrating them out, an one loop correction to this vertex can be obtained. In general, we can determine the effective action for reggeized gluons in terms of reggeon fields only. Inserting found classical solutions solutions in the action we will obtain some effective action for the reggeon fields $A_{+}$and $A_{-}$in the following form:

$\Gamma=\sum_{n, m=0}\left(A_{+}^{a_{1}} \cdots A_{+}^{a_{n}} K_{b_{1} \cdots b_{m}}^{a_{1} \cdots a_{n}} A_{-}^{b_{1}} \cdots A_{-}^{b_{m}}\right)$,

that will allow to calculate different reggeon-reggeon transition vertices $K_{b_{1} \cdots b_{m}}^{a_{1} \cdots a_{n}}$ responsible for the unitarization of the scattering amplitudes at high energies, which also can be used in calculations of the amplitudes of various processes with multi-Regge and quasi-multi-Regge kinematics. 
In general, it will be interesting to investigate possible relations of the considered framework with results of [6468 ] and we hope that further work in the proposed direction will allow to establish useful correspondences between the different small $x$ approaches and calculate high order corrections to the amplitudes of high-energy scattering.

Acknowledgements The authors are thankful to E.Levin and S. Pozdnyakov for helpful discussions concerning the subject of the paper and an especial thank to Jochen Bartels for his hospitality at Hamburg, where this project was initiated. L.L. acknowledges partial support of the Russian Scientific Fund project 14-2200281 and would like to thank the State University of St. Petersburg for the Grant SPSU 11.38.223.2015 and the Grant RFBI 16-02-01143 for support.

Open Access This article is distributed under the terms of the Creative Commons Attribution 4.0 International License (http://creativecomm ons.org/licenses/by/4.0/), which permits unrestricted use, distribution, and reproduction in any medium, provided you give appropriate credit to the original author(s) and the source, provide a link to the Creative Commons license, and indicate if changes were made. Funded by SCOAP . $^{3}$

\section{Appendix A: Induced current in the effective action}

In this Appendix we consider an $j^{+}$component of the induced current which can be obtained by variation of the current term in the effective action Eq. (1):

$j_{i n d}^{+}\left(v_{+}\right)=j_{-}^{i n d}\left(v_{+}\right)=\frac{1}{N} O\left(v_{+}\right)\left(\partial_{i}^{2} A^{+}\right) O^{T}\left(v_{+}\right)$.

The operators $O$ and $O^{T}$ are introduced in [2-7]:

$O=\partial_{+}\left(D_{+}^{-1}\right) ; \quad O^{T}=\left(D_{+}^{-1}\right) \overleftarrow{\partial}_{+}$

and have the following properties:

$\partial_{+} O=g v_{+} O, O^{T} \overleftarrow{\partial}_{+}=-g O^{T} v_{+}$

see Appendix B further. The Eq. (A.1) form of the current is general, the particular representations of the current in terms of P-exponentials, in turn, depend on the representations of $O$ operator, or, more precisely on the representation of the $\partial_{+}^{-1}$ operator. If we take the following simplest representation

$\frac{1}{\partial_{+}} f\left(x^{+}\right)=\int_{-\infty}^{x^{+}} \mathrm{d} x^{\prime} f\left(x^{\prime}\right)$,

we obtain for these operators:

$O=P e^{l g \int_{-\infty}^{x^{+}} \mathrm{d} x^{\prime}+v_{+}^{a} T_{a}}$ and

$O^{T}=P e^{l g \int_{x}^{\infty}+\mathrm{d} x^{\prime}+v_{+}^{a} T_{a}}$.

The variation of interaction term in the action can be calculated with the help of the formulas from Appendix B and results by induced current from Eq. (A.1):

$$
\begin{aligned}
& \delta\left(A_{+}\left(v_{+}\right) j_{\mathrm{reg}}^{+}\right) \\
& =-l\left(\delta v_{+}^{a}\right) \operatorname{tr}\left[T_{a} j_{\mathrm{ind}}^{+}\left(v_{+}\right)\right]=\left(\delta v_{+}^{a}\right) j_{a}^{+}\left(v_{+}\right) \\
& =-\frac{1}{N}\left(\delta v_{+}^{a}\right) \operatorname{tr}\left[T_{a} O T_{b} O^{T}\right]\left(\partial_{i}^{2} A_{b}^{+}\right),
\end{aligned}
$$

with $v_{+}=\imath T^{a} v_{+}^{a}$ representation of the gluon field used. In the case of adjoint representation ${ }^{5}$ we will obtain:

$$
\begin{aligned}
\delta\left(A_{+}\left(v_{+}\right) j_{\mathrm{reg}}^{+}\right) & =\left(\delta v_{+}^{a}\right) j_{a}^{+}\left(v_{+}\right) \\
& =\frac{1}{N}\left(\delta v_{+}^{a}\right) \operatorname{tr}\left[f_{a} O f_{b} O^{T}\right]\left(\partial_{i}^{2} A_{b}^{+}\right) \\
& =\frac{1}{N}\left(\delta v_{+}^{a}\right) U^{a b}\left(\partial_{i}^{2} A_{b}^{+}\right),
\end{aligned}
$$

that provides

$j_{a}^{+}\left(v_{+}=0\right)=-\partial_{i}^{2} A_{a}^{+}$.

The $U^{a b}$ exponential in Eq. (A.8) is the same as used in CGC approach of [14-20], see Eq. (26). The Eqs. (A.5)(A.6) forms of the operators can be modified in order to provide the action's unitarity at $x \rightarrow \pm \infty$. For that, the Eq. (A.4) operator can be modified as: ${ }^{6}$

$\frac{1}{\partial_{+}} f\left(x^{+}\right)=\frac{1}{2} \int \mathrm{d} x^{\prime+} \epsilon\left(x^{+}-x^{\prime}\right) f\left(x^{\prime}\right)$,

where $\epsilon\left(x^{+}-x^{\prime}\right)$ is a sign function, that corresponds to the different from Eq. (A.4) definition of the integral operator $\partial_{+}^{-1}$, where the regularization of the corresponding $1 / k_{+}$ pole in momentum space must be understood as principal value prescription, see details in [2-7]. In this case, more complicated expressions for the operators will be obtained, see also Appendix B below.

\footnotetext{
$\overline{5}$ The general form of the current does not depend on the representation, in our particular case we take $\left(T_{a}\right)_{b c}=-\imath f_{a b c}$ in the current, representing only "external" indexes in the expression.

${ }^{6}$ We use the Kogut-Soper convention for the metric tensor, in the Lepage-Brodsky convention there is $1 / 4$ coefficient in the front of the following expression.
} 


\section{Appendix B: Representation and properties of operators $O$ and $O^{T}$}

For the arbitrary representation of gauge field $v_{+}=\imath T^{a} v_{+}^{a}$ with $D_{+}=\partial_{+}-g v_{+}$, we can consider the following representation of $O$ and $O^{T}$ operators: $^{7}$

$O_{x}=\delta^{a b}+g \int \mathrm{d}^{4} y G_{x y}^{+a a_{1}}\left(v_{+}(y)\right)_{a_{1} b}=1+g G_{x y}^{+} v_{+y}$

and correspondingly

$O_{x}^{T}=1+g v_{+y} G_{y x}^{+}$,

which is redefinition of the operator expansions used in [2-7] in terms of Green's function instead integral operators, see Appendix A above. The Green's function in above equations we understand as Green's function of the $D_{+}$operator and express it in the perturbative sense as:

$G_{x y}^{+}=G_{x y}^{+0}+g G_{x z}^{+0} v_{+z} G_{z y}^{+}$

and

$G_{y x}^{+}=G_{y x}^{+0}+g G_{y z}^{+} v_{+z} G_{z x}^{+0}$,

with the bare propagators defined as (there is no integration on index $x$ in expressions)

$\partial_{+x} G_{x y}^{+0}=\delta_{x y}, \quad G_{y x}^{+0} \overleftarrow{\partial}_{+x}=-\delta_{x y}$

The following properties of the operators now can be derived:

1. $\delta G_{x y}^{+}=g G_{x z}^{+0}\left(\delta v_{+z}\right) G_{z y}^{+}+G_{x z}^{+0} v_{+z} \delta G_{z y}^{+}$

$$
\begin{aligned}
& =g G_{x z}^{+0}\left(\delta v_{+z}\right) G_{z y}^{+}+G_{x z}^{+0} v_{+z}\left(\delta G_{z p}^{+}\right) D_{+p} G_{p y}^{+} \\
& =g\left(G_{x z}^{+0}\left(\delta v_{+z}\right) G_{z y}^{+}-G_{x z}^{+0} v_{+z} G_{z p}^{+}\left(\delta D_{+p}\right) G_{p y}^{+}\right) \\
& =g\left(G_{x p}^{+0}+G_{x z}^{+0} v_{+z} G_{z p}^{+}\right) \delta v_{+p} G_{p y}^{+} \\
& =g G_{x p}^{+} \delta v_{+p} G_{p y}^{+} ;
\end{aligned}
$$

$$
\text { 2. } \begin{aligned}
\delta O_{x} & =g G_{x y}^{+}\left(\delta v_{+y}\right)+g\left(\delta G_{x y}^{+}\right) v_{+y} \\
& =g G_{x p}^{+} \delta v_{+p}\left(1+g G_{p y}^{+} v_{+y}\right) \\
& =g G_{x p}^{+} \delta v_{+p} O_{p} ;
\end{aligned}
$$

\footnotetext{
7 Due the light cone gauge we consider here only $O\left(x^{+}\right)$operators. The construction of the representation of the $O\left(x^{-}\right)$operators can be done similarly. We also note, that the integration is assumed for repeating indexes in expressions below if it is not noted otherwise.
}

3. $\partial_{+x} \delta O_{x}=g\left(\partial_{+x} G_{x p}^{+}\right) \delta v_{+p} O_{p}$

$$
\begin{aligned}
& =g\left(1+g v_{+x} G_{x p}^{+}\right) \delta v_{+p} O_{p} \\
& =g O_{x}^{T} \delta v_{+x} O_{x} ;
\end{aligned}
$$

4. $\partial_{+x} O_{x}=g\left(\partial_{+x} G_{x y}^{+}\right) v_{+y}$

$$
=g v_{+x}\left(1+g G_{x y}^{+} v_{+y}\right)=g v_{+x} O_{x} ;
$$

5. $O_{x}^{T} \overleftarrow{\partial}_{+x}=g v_{+y}\left(G_{y x}^{+} \overleftarrow{\partial}_{+x}\right)$

$$
=-g\left(1+v_{+y} G_{y x}^{+}\right) v_{+x}=-g O_{x}^{T} v_{+x} .
$$

We see, that the operator $O$ and $O^{T}$ have the properties of ordered exponents. For example, choosing bare propagators as

$G_{x y}^{+0}=\theta\left(x^{+}-y^{+}\right) \delta_{x y}^{3}, \quad G_{y x}^{+0}=\theta\left(y^{+}-x^{+}\right) \delta_{x y}^{3}$,

we immediately reproduce:

$O_{x}=P e^{g \int_{-\infty}^{x^{+}} \mathrm{d} x^{\prime}+v_{+}\left(x^{\prime}\right)}, \quad O_{x}^{T}=P e^{g \int_{x^{+}}^{\infty} \mathrm{d} x^{\prime+} v_{+}\left(x^{\prime+}\right)}$.

The form of the bare propagator $G_{x y}^{+0}=\frac{1}{2}\left[\theta\left(x^{+}-y^{+}\right)\right.$ $\left.-\theta\left(y^{+}-x^{+}\right)\right] \delta_{x y}^{3}$ which correspond to the Eq. (A.10) integral operator will lead to the more complicated representations of $O$ and $O^{T}$ operators, see in [2-7].

Now we consider a variation of the action's full current:

$$
\begin{aligned}
\delta \operatorname{tr}\left[v_{+x} O_{x} \partial_{i}^{2} A^{+}\right] & =\frac{1}{g} \delta \operatorname{tr}\left[\left(\partial_{+x} O_{x}\right) \partial_{i}^{2} A^{+}\right] \\
& =\frac{1}{g} \operatorname{tr}\left[\left(\partial_{+x} \delta O_{x}\right) \partial_{i}^{2} A^{+}\right] \\
& =\operatorname{tr}\left[O_{x}^{T} \delta v_{+x} O_{x}\left(\partial_{i}^{2} A^{+}\right)\right],
\end{aligned}
$$

which can be rewritten in the familiar form used in the paper:

$$
\begin{aligned}
\delta\left(v_{+} J^{+}\right) & =\delta \operatorname{tr}\left[\left(v_{+x} O_{x} \partial_{i}^{2} A^{+}\right)\right] \\
& =-\delta v_{+}^{a} \operatorname{tr}\left[T_{a} O T_{b} O^{T}\right]\left(\partial_{i}^{2} A_{b}^{+}\right) .
\end{aligned}
$$

We also note, that with the help of Eq. (B.1) representation of the $O$ operator the full action's current can we written as follows

$$
\begin{aligned}
& \operatorname{tr}\left[\left(v_{+x} O_{x}-A_{+}\right) \partial_{i}^{2} A^{+}\right] \\
& \quad=\operatorname{tr}\left[\left(v_{+}-A_{+}+v_{+x} G_{x y}^{+} v_{+y}\right)\left(\partial_{i}^{2} A^{+}\right)\right] .
\end{aligned}
$$




\section{Appendix C: NLO solution of equations of motion}

In this Appendix we derive expressions for the next order solution of the equations of motion. We write the longitudinal field of interests as

$v_{+}^{a}=A_{+}^{a}+g v_{+1}^{a}\left(x_{\perp}, x^{-}, x^{+}\right)$,

and the transverse field in the next order approximation as:

$v_{i}^{a}=v_{i 0}^{a}+g v_{i 1}^{a}\left(x_{\perp}, x^{-}, x^{+}\right)+g \tilde{v}_{i 1}^{a}\left(x_{\perp}, x^{-}, x^{+}\right)$

with the following constraint

$\partial^{i} v_{i 1}^{a}=0$

where

$v_{i 0}^{a}=\rho_{i}^{b}\left(x_{\perp}, x^{-}\right) U^{a b}\left(A_{+}\right)$.

1. Let's consider again the equations of motion and will begin from Eq. (12):

$-\left(D_{i}\left(\partial_{-} v^{i}\right)\right)_{a}-\partial_{-}^{2} v_{a+}=j_{a}^{+}\left(A_{+}\right)$

which at requested order has the following form:

$$
\begin{aligned}
& -g \partial^{i} \partial_{-} \tilde{v}_{i 1}^{a}-\left(\partial^{i} U^{a b}\right)\left(\partial_{-} \rho_{i}^{b}\right) \\
& -g f_{a b c}\left(U^{b b^{\prime}} \rho^{i b^{\prime}}\right)\left(U^{c c^{\prime}}\left(\partial_{-} \rho_{i}^{c^{\prime}}\right)\right) \\
& -g \partial_{-}^{2} v_{+1}^{a}=0 .
\end{aligned}
$$

Denoting

$$
g j_{a 1}^{+}=g f_{a b c}\left(U^{b b^{\prime}} \rho^{i b^{\prime}}\right)\left(U^{c c^{\prime}}\left(\partial_{-} \rho_{i}^{c^{\prime}}\right)\right),
$$

we obtain:

$v_{+1}^{a}=-\partial^{i} \partial_{-}^{-1} \tilde{v}_{i 1}^{a}-\frac{1}{g}\left(\partial^{i} U^{a b}\right)\left(\partial_{-}^{-1} \rho_{i}^{b}\right)-\left(\partial_{-}^{-2} j_{a 1}^{+}\right)$,

or

$$
\partial^{i} \tilde{v}_{i 1}^{a}=-\partial_{-} v_{+1}^{a}-\frac{1}{g}\left(\partial^{i} U^{a b}\right) \rho_{i}^{b}-\left(\partial_{-}^{-1} j_{a 1}^{+}\right) .
$$

2. Now we consider equation of motion Eq. (17)

$$
\begin{aligned}
& \left(D_{+}\left[v_{+}\right]\left(\partial_{-} v_{+}\right)\right)_{a}+\left(D^{i}\left(\partial_{i} v_{+}\right)\right)_{a} \\
& -\left(D^{i}\left[v_{i}\right]\left(D_{+}\left[v_{+}\right] v_{i}\right)\right)_{a}=-\partial_{i}^{2} A_{a+}
\end{aligned}
$$

which at NLO reads as

$$
\begin{gathered}
\partial_{+} \partial_{-} v_{+1}^{a}+\partial_{i} \partial^{i} v_{+1}^{a}-\partial_{+} \partial^{i} \tilde{v}_{i 1}^{a} \\
+f_{a b c} U^{b b^{\prime}} \rho_{b^{\prime}}^{i} \partial_{i} A_{+}^{c}=0 .
\end{gathered}
$$

or

$$
\begin{gathered}
\partial_{+} \partial_{-} v_{+1}^{a}+\partial_{i} \partial^{i} v_{+1}^{a}-\partial_{+} \partial^{i} \tilde{v}_{i 1}^{a} \\
+\frac{1}{g}\left(\partial_{+} \partial^{i} U^{a b}\right) \rho_{i}^{b}=0 .
\end{gathered}
$$

Inserting Eq. (C.9) into Eq. (C.11) one obtains

$$
\begin{gathered}
\left(2 \partial_{+} \partial_{-}+\partial_{i} \partial^{i}\right) v_{+1}^{a}=\square v_{+1}^{a}=-\frac{1}{g}\left(\partial_{+} \partial^{i} U^{a b}\right) \rho_{i}^{b} \\
-f_{a b c} U^{b b^{\prime}} \rho_{b^{\prime}}^{i} \partial_{i} A_{+}^{c}-\left(\partial_{+} \partial_{-}^{-1} j_{a 1}^{+}\right),
\end{gathered}
$$

that gives

$v_{+1}^{a}=-\frac{2}{g} \square^{-1}\left(\left(\partial_{+} \partial^{i} U^{a b}\right) \rho_{i}^{b}\right)-\square^{-1}\left(\partial_{+} \partial_{-}^{-1} j_{a 1}^{+}\right)$

Taking into account that the last term in Eq. (C.14) is of order $g^{2}$, finally for this field we have:

$v_{+1}^{a}=-\frac{2}{g} \square^{-1}\left(\left(\partial_{+} \partial^{i} U^{a b}\right) \rho_{i}^{b}\right)$.

In turn, inserting Eqs. (C.8) into (C.12), one obtains

$$
\begin{gathered}
2 \partial_{+}\left(\partial^{j} \tilde{v}_{j 1}^{a}\right)+\partial_{j} \partial^{j}\left(\partial_{-}^{-1} \partial^{i} \tilde{v}_{i 1}^{a}\right) \\
+\frac{1}{g} \partial_{j} \partial^{j}\left(\left(\partial^{i} U^{a b}\right)\left(\partial_{-}^{-1} \rho_{i}^{b}\right)\right) \\
+\left(\partial_{+} \partial_{-}+\partial_{j} \partial^{j}\right)\left(\partial_{-}^{-2} j_{a 1}^{+}\right)=0,
\end{gathered}
$$

which we rewrite as

$$
\begin{aligned}
& \square\left(\partial^{i} \tilde{v}_{i 1}^{a}\right) \\
& =-\frac{1}{g} \partial_{j} \partial^{j}\left(\left(\partial^{i} U^{a b}\right) \rho_{i}^{b}\right)-\left(\partial_{+} j_{a 1}^{+}+\partial_{j} \partial^{j} \partial_{-}^{-1} j_{a 1}^{+}\right) .
\end{aligned}
$$

Therefore, with NLO precision, the answer is

$$
\tilde{v}_{i 1}^{a}=-\frac{1}{g} \square^{-1} \partial_{i}\left(\left(\partial^{j} U^{a b}\right) \rho_{j}^{b}\right)-\square^{-1}\left(\partial_{i} \partial_{-}^{-1} j_{a 1}^{+}\right) .
$$


3. For the last equation, Eq. (20),

$$
2\left(D_{+}\left(\partial_{-} V_{i}\right)\right)_{a}-\left(D_{i}\left(\partial_{-} V_{+}\right)\right)_{a}+\left(D^{j} G_{j i}\right)_{a}=0
$$

at NLO we write

$$
\begin{array}{rr}
2 \partial_{+} \partial_{-} v_{i 1}^{a}+2 \partial_{+} \partial_{-} \tilde{v}_{i 1}^{a}+\partial_{j}^{2} v_{i 1}^{a}+\left(\partial_{j}^{2} \tilde{v}_{i 1}^{a}-\partial_{i} \partial^{j} \tilde{v}_{j 1}^{a}\right) \\
+\partial^{j} F_{j i}^{a}-\partial_{i} \partial_{-} v_{+1}^{a}=0,
\end{array}
$$

with $\partial^{j} F_{j i}^{a}$ function as remaining NLO part of $D^{j} G_{j i}$ which depends on $\rho_{i}$ and $A_{+}$fields only. Therefore

$$
v_{i 1}^{a}=-\square^{-1}\left(\partial^{j} F_{j i}^{a}\right)
$$

with the same Eq. (C.17) for the $\tilde{v}_{i 1}^{a}$ function. The complete NLO correction to $v_{i}^{a}$ function reads as

$$
\begin{aligned}
V_{i 1}^{a} & =v_{i 1}^{a}\left(x_{\perp}, x^{-}, x^{+}\right)+\tilde{v}_{i 1}^{a}\left(x_{\perp}, x^{-}, x^{+}\right) \\
& =-\square^{-1}\left(\partial^{j} F_{j i}^{a}+\frac{1}{g} \partial_{i}\left(\left(\partial^{j} U^{a b}\right) \rho_{j}^{b}\right)+\partial_{i} \partial_{-}^{-1} j_{a 1}^{+}\right) .
\end{aligned}
$$

\section{References}

1. V.N. Gribov, Sov. Phys. JETP 26, 414 (1968)

2. L.N. Lipatov, Nucl. Phys. B 452, 369 (1995)

3. L.N. Lipatov, Phys. Rep. 286, 131 (1997)

4. L.N. Lipatov, Subnucl. Ser. 49, 131 (2013)

5. L.N. Lipatov, Int. J. Mod. Phys. Conf. Ser. 39, 1560082 (2015)

6. L.N. Lipatov, Int. J. Mod. Phys. A 31(28/29), 1645011 (2016)

7. L.N. Lipatov, EPJ Web Conf. 125, 01010 (2016)

8. L.N. Lipatov, Sov. J. Nucl. Phys. 23, 338 (1976). [Yad. Fiz. 23, 642 (1976)]

9. E.A. Kuraev, L.N. Lipatov, V.S. Fadin, Sov. Phys. JETP 45, 199 (1977)

10. E.A. Kuraev, L.N. Lipatov, V.S. Fadin, Zh Eksp, Teor. Fiz. 72, 377 (1977)

11. I.I. Balitsky, L.N. Lipatov, Sov. J. Nucl. Phys. 28, 822 (1978)

12. I.I. Balitsky, L.N. Lipatov, Yad. Fiz. 28, 1597 (1978)

13. L. McLerran, R. Venugopalan, Phys. Rev. D 49, 2233 (1994). [Phys. Rev. D 49, 3352 (1994)]

14. J. Jalilian-Marian, A. Kovner, L. McLerran, H. Weigert, Phys. Rev. D 55, 5414 (1997)

15. J. Jalilian-Marian, A. Kovner, A. Leonidov, H. Weigert, Nucl. Phys. B 504, 415 (1997)

16. J. Jalilian-Marian, A. Kovner, A. Leonidov, H. Weigert, Phys. Rev. D 59, 014014 (1998)

17. J. Jalilian-Marian, A. Kovner, H. Weigert, Phys. Rev. D 59, 014015 (1998)

18. E. Iancu, A. Leonidov, L.D. McLerran, Nucl. Phys. A 692, 583 (2001)

19. E. Iancu, A. Leonidov, L.D. McLerran, Phys. Lett. B 510, 133 (2001)
20. E. Ferreiro, E. Iancu, A. Leonidov, L. McLerran, Nucl. Phys. A 703, 489 (2002)

21. L.V. Gribov, E.M. Levin, M.G. Ryskin, Phys. Rep. 100, 1 (1983)

22. Y. Hatta, Nucl. Phys. A 768, 222 (2006)

23. Y. Hatta, Nucl. Phys. A 781, 104 (2007)

24. Y.M. Makeenko, A.A. Migdal, Phys. Lett. 88B, 135-137 (1979)

25. U. Heinz, Phys. Rev. Lett. 51, 351 (1983)

26. U. Heinz, Phys. Lett. B 144, 228 (1984)

27. U. Heinz, Ann. Phys. 161, 48 (1985)

28. U. Heinz, Ann. Phys. 168, 148 (1986)

29. U. Heinz, Z. Phys. C 38, 203 (1988)

30. U. Heinz, Phys. A 158, 111 (1989)

31. J. Jalilian-Marian, S. Jeon, R. Venugopalan, J. Wirstam, Phys. Rev. D 62, 045020 (2000)

32. J. Jalilian-Marian, S. Jeon, R. Venugopalan, Phys. Rev. D 63, 036004 (2001)

33. J.P. Blaizot, E. Iancu, Phys. Rep. 359, 355 (2002)

34. D.F. Litim, C. Manuel, Phys. Rep. 364, 451 (2002)

35. I. Balitsky, Nucl. Phys. B 463, 99 (1996)

36. Y.V. Kovchegov, Phys. Rev. D 60, 034008 (1999)

37. Y.V. Kovchegov, Phys. Rev. D 61, 074018 (2000)

38. M.A. Braun, Phys. Lett. B 483, 115 (2000)

39. M.A. Braun, Eur. Phys. J. C 33, 113 (2004)

40. S. Bondarenko, M.A. Braun, Nucl. Phys. A 799, 151 (2008)

41. J. Bartels, S. Bondarenko, K. Kutak, L. Motyka, Phys. Rev. D 73, 093004 (2006)

42. S. Bondarenko, L. Motyka, Phys. Rev. D 75, 114015 (2007)

43. S. Bondarenko, Phys. Lett. B 665, 72 (2008)

44. S. Bondarenko, Nucl. Phys. A 853, 71 (2011)

45. D. Amati, L. Caneschi, R. Jengo, Nucl. Phys. B 101, 397 (1975)

46. R. Jengo, Nucl. Phys. B 108, 447 (1976)

47. M. Ciafaloni, Nucl. Phys. B 146, 427 (1978)

48. E. Levin, A. Prygarin, Eur. Phys. J. C 53, 385 (2008)

49. S. Bondarenko, Eur. Phys. J. C 71, 1587 (2011)

50. S. Bondarenko, L. Horwitz, J. Levitan, A. Yahalom, Nucl. Phys. A 912, 49 (2013)

51. L.N. Lipatov, Nucl. Phys. Proc. Suppl. 99A, 175 (2001)

52. M.A. Braun, M.I. Vyazovsky, Eur. Phys. J. C 51, 103 (2007)

53. M.A. Braun, M.Y. Salykin, M.I. Vyazovsky, Eur. Phys. J. C 65, $385(2010)$

54. M.A. Braun, L.N. Lipatov, M.Y. Salykin, M.I. Vyazovsky, Eur. Phys. J. C 71, 1639 (2011)

55. M.A. Braun, M.Y. Salykin, M.I. Vyazovsky, Eur. Phys. J. C 72, $1864(2012)$

56. M. Hentschinski, A. Sabio Vera, Phys. Rev. D 85, 056006 (2012)

57. M.A. Braun, M.Y. Salykin, S.S. Pozdnyakov, M.I. Vyazovsky, Eur. Phys. J. C 72, 2223 (2012)

58. J. Bartels, L.N. Lipatov, G.P. Vacca, Phys. Rev. D 86, 105045 (2012)

59. M.A. Braun, S.S. Pozdnyakov, M.Y. Salykin, M.I. Vyazovsky, Eur. Phys. J. C 73(9), 2572 (2013)

60. G. Chachamis, M. Hentschinski, J.D. Madrigal Martnez, A. Sabio Vera, Phys. Part. Nucl. 45(4), 788 (2014)

61. J. Bartels, Nucl. Phys. B 175, 365 (1980)

62. J. Kwiecinski, M. Praszalowicz, Phys. Lett. B 94, 413 (1980)

63. J. Bartels, V.S. Fadin, L.N. Lipatov, G.P. Vacca, Nucl. Phys. B 867, 827 (2013)

64. I. Balitsky, Phys. Rev. D 72, 074027 (2005)

65. Y. Hatta, E. Iancu, L. McLerran, A. Stasto, D.N. Triantafyllopoulos, Nucl. Phys. A 764, 423 (2006)

66. I. Balitsky, Phys. Rev. D 60, 014020 (1999)

67. I. Balitsky, in At the frontier of particle physics, vol. 2, ed. by M. Shifman (2002) pp. 1237-1342

68. I. Balitsky, Nucl. Phys. B 629, 290 (2002) 